\title{
Permission for tobacco-smoking in the Health Services, according to the Psychiatric Population and the General Population
}

\section{Renata Marques de Oliveira, Jair Lício Ferreira Santos, Antonia R. Ferreira Furegato Universidade de São Paulo}

\section{Introduction}

The prevalence of smokers in the psychiatric population is a public health problem. This study intended to investigate the opinion about smoking prohibition.

\section{Objective}

To investigate the opinion of the psychiatric population and the general population regarding prohibition of tobacco-smoking in the health services.

\section{Materials and methods}

A cross-sectional epidemiological study undertaken in Brazil. A total of 378 persons participated, from three health services: Mental Health Outpatient Center (MHOC, $n=126)$, Psychiatric Hospital $(\mathrm{PH}, \mathrm{n}=126)$ and Primary Healthcare Center (PHC, $n=126)$.

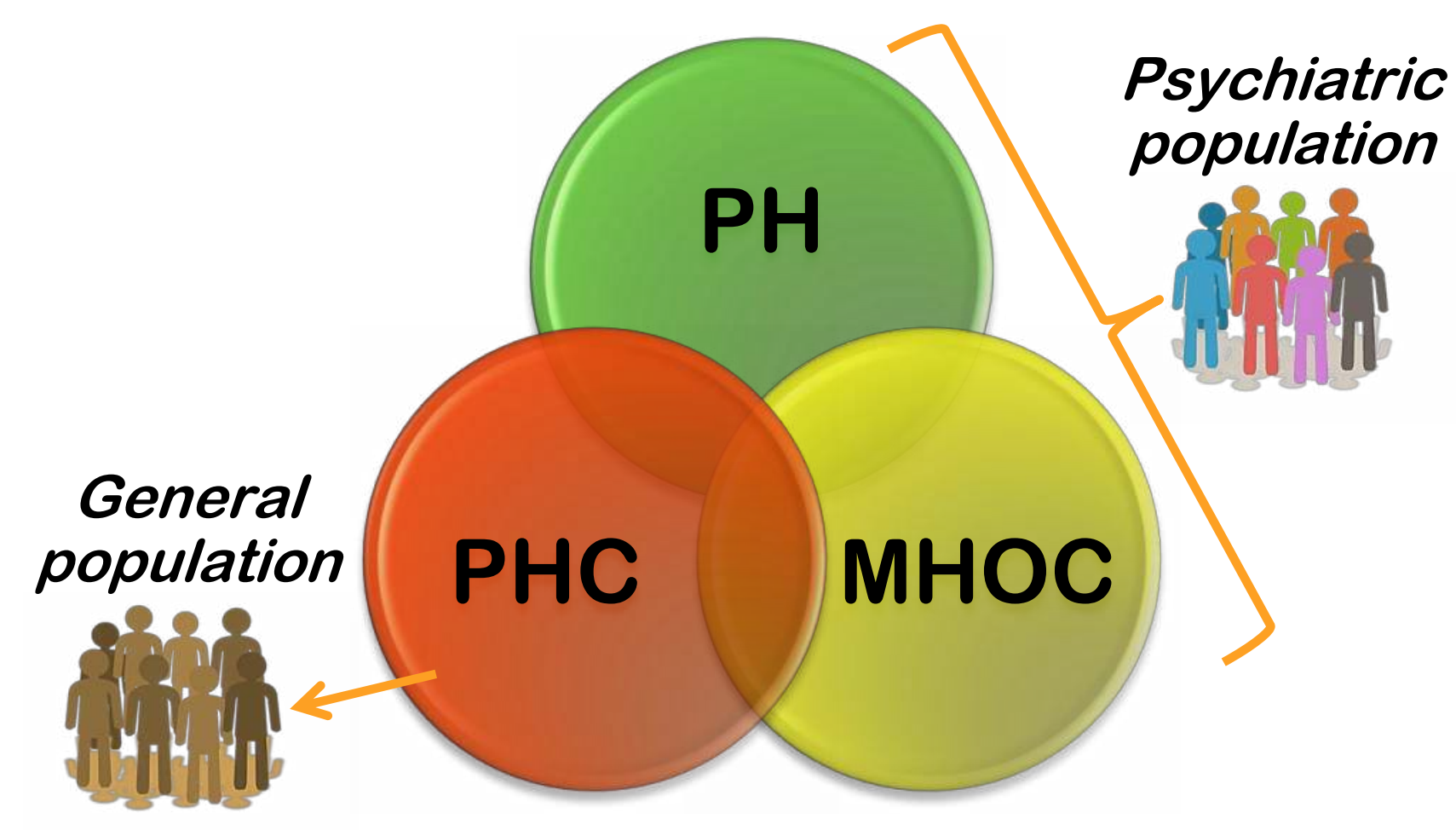

The data, obtained through individual interviews, was analyzed through descriptive statistics and logistic regression.

\section{Results}

$35.4 \%$ were smokers $(\mathrm{MHOC}=27 \%, \mathrm{PH}=60 \%$, $\mathrm{PHC}=19 \%)$.
The subjects from the hospital favored the prohibition of tobacco-smoking less than people attended in the extra-hospital units (MHOC=84\%, PH=69\%, PHC=100\%).

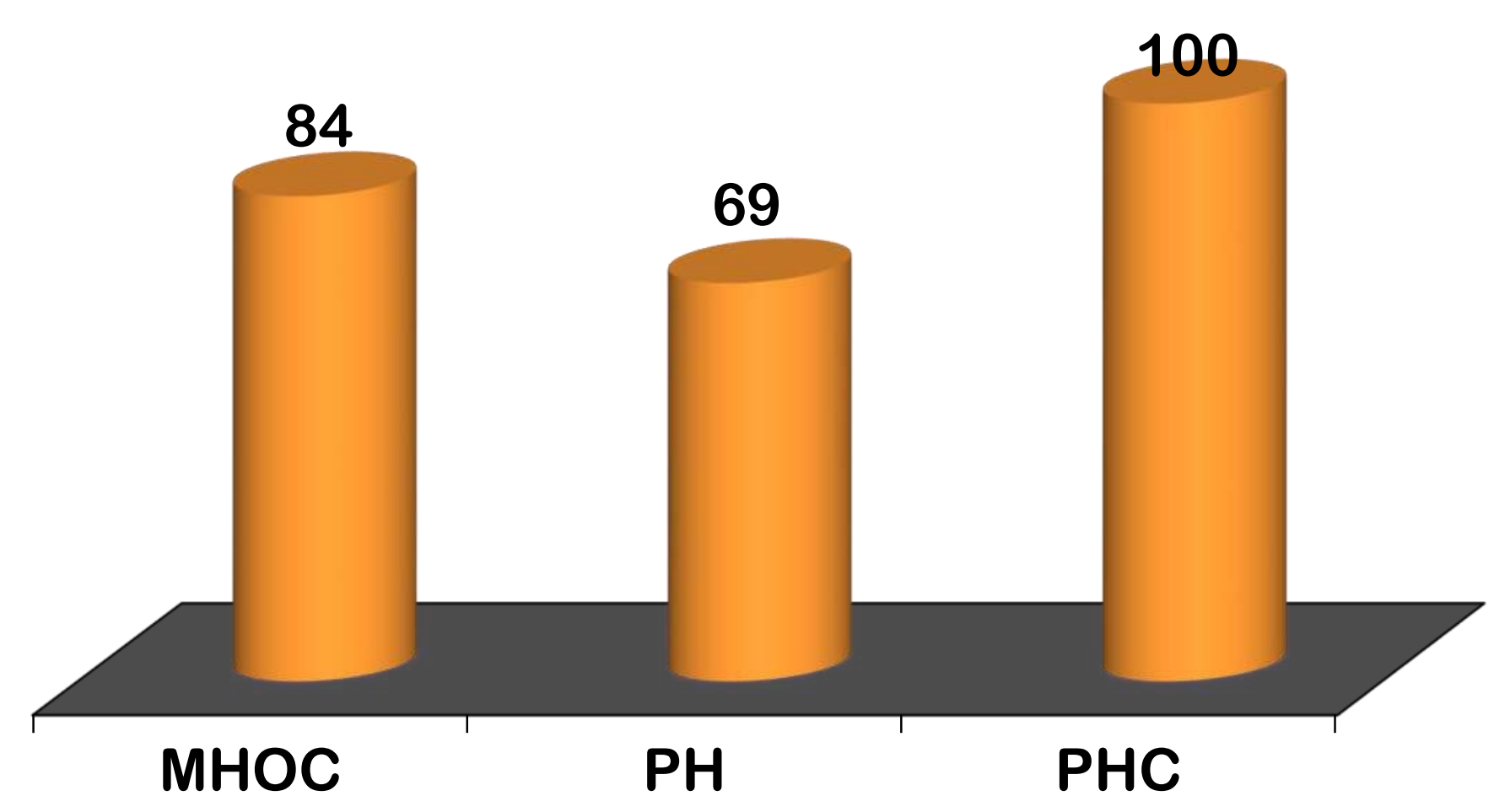

This difference is explained by the greater number of smokers in the $\mathrm{PH}$ and because this is the historical/cultural heir of the old lunatic asylums.

People with a history of four or more admissions $(O R=3.24)$ and the smokers $(O R=3.18)$, were those who most agreed that patients have the right to smoke in health services. Consistently, they were the least likely to agree that smoking should be prohibited in the health services $(O R=0.18$ and $O R=0.26$, respectively).

\section{Conclusion}

The psychiatric population, particularly that of the psychiatric hospital, was less tolerant to the smoking prohibition, reflecting the tobacco culture in this type of institution. Knowing the psychiatric population's and general population's opinion on this issue is important for the professionals' work, as this is a current and polemic topic. 